nominates the steering committee members and a plenary meeting to be kept informed about research they are involved in.

2) Insight Panels:

Access to technology was viewed as the major barrier to remote involvement. Mechanisms to overcome included multiple modes of communication: online, tele phone, postal communication. Providing the opportunity for face-to-face or speak ing directly with a researcher in an informal setting was seen as crucial in building interpersonal relationships and sustaining involvement.

3) Patient Educator:

Extremely well received. Barriers to participation revolved largely around travel and physical accessibility. This can be overcome with in-house resources.

Our research strategy is being revised with the PPI strategy as a central tenet. We are adopting the new steering committee under the three-tier format and yearly research meeting with plenary session. We have a PPI newsletter, the editorial board for which is made up of researchers and patient insight partners. A patient educator module is under review for incorporation into a new PhD programme. We have expanded our research into multidisciplinary areas, with new sociology researchers and psychology collaborators.

Conclusions: The development a true patient partnership in our group has fundamentally changed the scope and remit of our research, allowing us to expand our biomedical and clinical research into a more holistic model.

Disclosure of Interest: None declared

DOI: 10.1136/annrheumdis-2018-eular.6815

\section{OP0210-PARE DUTCH JUVENILE IDIOPATHIC ARTHRITIS PATIENTS, CARERS AND CLINICIANS CREATING A RESEARCH AGENDA TOGETHER FOLLOWING THE JAMES LIND ALLIANCE METHOD}

C.G Schoemaker ${ }^{1,2}$, W. Armbrust ${ }^{3,4}$, J.F. Swart ${ }^{2,4}$, E. Versluis $^{1}$, W. Olsder ${ }^{5}$, N M. Wulffraat ${ }^{2} .{ }^{1}$ Dutch JIA patient organisation, member of ENCA, Rijen;

${ }^{2}$ Department of Pediatric Rheumatology, University Medical Center Utrecht, Utrecht, ${ }^{3}$ Department of Pediatric Rheumatology, University Medical Center Groningen, Groningen; ${ }^{4}$ Netherlands Association for Pediatric Rheumatology, Utrecht, ${ }^{5}$ Youth-R-Well.com, Young Patient Organization, Lisse, Netherlands

Background: Biomedical research should support patients, carers and clinicians to take important decisions in the consulting room and eventually to improve the lives of patients. 'Thus far the end-users of Juvenile Idiopathic Arthritis (JIA) research have not been involved in the prioritisation of future research. The JIA research community clearly sees the unmet need and has repeatedly expressed the wish to do something about this. As Parsons et al. have put it: 'Understanding young people's research priorities is important to develop research that is in tune with their needs'. 'P Putting this into practice starts with a search for relevant issues working together with the end users of scientific knowledge on JIA - patients, carers and clinicians - and to prioritise research questions that can really make a difference.

Objectives: In 2018 Dutch organisations of patients, carers and clinicians will collaboratively develop a research agenda for JIA, following the James Lind Alliance methodology. ${ }^{3,4}$ An established research agenda, created by patients, carers and clinicians, will inform researchers and research funders about what the most important, relevant research questions for JIA are.

Methods: The James Lind Alliance (JLA) methodology enables us to do a sys tematic search for unanswered questions that are relevant to patients, carers and clinicians. 'In a 'Priority Setting Partnership' (PSP), we will gradually establish a top 10 list of the most important unanswered research questions for JIA. In this process the input from patients and their carers will be given the same weight as that from clinicians.

The Dutch JIA PSP will be led by a steering group. This steering group coordinates the PSP and organises the activities. It will include representatives of patients (for JIA: young and adult JIA-patients), carers (for JIA: parents and spouses) and clinicians (for JIA: paediatric rheumatologists, physiotherapists, nurses, psychologists, social workers, ophthalmologist, etc.).

Results: The Dutch JIA organisations support the agenda; also financially. Following the JLA method it will take approximately twelve to eighteen months to for mulate a research agenda, so te research agenda for JIA will be published in 2019.
Conclusions: A research agenda that will be jointly inspired by patients. carers and clinicians can really make a difference for decision-making in the consulting room and for the lives of JIA-patients.

\section{REFERENCES}

[1] Macleod MR, Michie S, Roberts I, et al. Biomedical research: increasing value, reducing waste. Lancet 2014;383:101-4. doi:10.1016/S0140-6736 (13)62329-6

[2] Parsons S, Thomson W, Cresswell K, Starling B, McDonagh JE. Barbara Ansell, National Network for Adolescent Rheumatology. What do young people with rheumatic disease believe to be important to research about their condition? A UK-wide study. Pediatr Rheumatol Online J 2017;15:53.

[3] Schoemaker CG, Prakken AB, Furth EF. Patients and physicians creating a research agenda together: the method of the British James Lind Alliance [Article in Dutch]. Ned Tijdschr Geneeskd 2017;161(0):D1764.

[4] JLA Guidebook. www.jla.nihr.ac.uk/jla-guidebook [Accessed 24 Jan 2018].

Disclosure of Interest: None declared

DOI: 10.1136/annrheumdis-2018-eular.2756

\section{THURSDAY, 14 JUNE 2018}

\section{Can we improve the care of gout?}

\section{OP0211 ULTRASOUND SHOWS RAPID REDUCTION OF URIC ACID LOAD DURING A TREAT-TO-TARGET APPROACH IN GOUT PATIENTS: RESULTS FROM A LONGITUDINAL STUDY (NOR-GOUT)}

${ }_{\text {H.B. Hammer }}{ }^{1}$, L. Karoliussen ${ }^{1}$, L. Terslev ${ }^{2}$, E.A. Haavardsholm ${ }^{1,3}$, T.K. Kvien ${ }^{1}$, T. Uhlig ${ }^{1}{ }^{1}$ Dept Of Rheumatology, Diakonhjemmet hosptial, Oslo, Norway, ${ }^{2}$ Centre for Rheumatology and Spinal Diseases, Copenhagen University Hospital at Glostrup, Copenhagen, Denmark; ${ }^{3}$ University of Oslo, Oslo, Norway

Background: Ultrasound (US) has received an increasing attention in detecting uric monosodium urate (MSU) deposits, and is included as a domain in the ACR/ EULAR classification criteria for gout. The OMERACT US group has developed definitions for US elementary lesions in gout including the double contour sign (DC) (deposits of crystals on the surface of cartilage), tophus (larger hypo-echoic aggregation of crystals, usually well delineated), aggregates (small hyper-echoic deposits) and erosions. MSU deposits may be found in many different regions with some predilection sites, but only a few small studies have explored the decrease of deposits during treatment.

Objectives: To explore by US the longitudinal resolution of MSU deposits during a treat-to-target approach with urate lowering therapy (ULT) in patients with gout.

Methods: In a prospective observational study, patients with crystal-proven gout were included if they presented after a recent gout flare and had increased serum urate levels (>360 $\mu \mathrm{mol} / \mathrm{L} />6 \mathrm{mg} / \mathrm{dl}$ ). In a treat-to-target approach using ULT and increasing drug doses with monthly follow-up until treatment target was met $(<360$ $\mu \mathrm{mol} / \mathrm{L}$, or $<300 \mu \mathrm{mol} / \mathrm{L}$ if clinical tophi). An extensive US assessment was performed (GE E9 machine, grey scale $15 \mathrm{MHz}$ ) at baseline and after 3, 6 and 12 months to detect MSU deposits, using the OMERACT definitions for DC, toph and aggregates with bilateral assessment of radiocarpal joints, MCP 2, insertion of triceps and quadriceps, proximal and distal patellar tendon, the Achilles tendon and cartilage of distal femur (maximal flexed knee) and the talar cartilage of tibiotalar joint and MTP 1 joint. The degree of elementary lesions was semi-quantitatively scored $0-3$ ( 0 =none, $1=$ possible, $2=$ certain, $3=$ major deposits). Sum scores of DC, tophi and aggregates, as well as total sum score of all lesions, were calcu lated for each visit. Changes from baseline were explored by paired samples Ttest and response by Standardised Response Mean (SRM).

Results: 161 patients were included at baseline (93.3\% men, mean (SD) age 57.0 (14.1) years, disease duration 8.0 (7.7) years). The mean (SD) serum urate level decreased from 487 (82) $\mu \mathrm{mol} / \mathrm{L}$ at baseline to $312(52) \mu \mathrm{mol} / \mathrm{L}$ at 12 months, with $72 \%$ reaching the target at 3 months, and $84 \%$ at 12 months. Sum scores of deposits decreased over 12 months (table 1 , with ${ }^{*}=p<0.05,{ }^{* *}=p<0.001$ ), and the numeric decrease was largest for DC (figure 1). SRM from baseline to 3, 6 and 12 months was $0.73,1.02$ and 1.26 for DC, $0.06,0.57$ and 0.91 for tohpi and 0.20 0.51 and 0.66 for aggregates. 